\title{
Kohn-Luttinger Superconductivity in Twisted Bilayer Graphene
}

\author{
J. González ${ }^{1}$ and T. Stauber ${ }^{2}$ \\ ${ }^{1}$ Instituto de Estructura de la Materia, CSIC, E-28006 Madrid, Spain \\ ${ }^{2}$ Materials Science Factory, Instituto de Ciencia de Materiales de Madrid, CSIC, E-28049 Madrid, Spain
}

(Received 2 July 2018; published 18 January 2019)

\begin{abstract}
We show that the recently observed superconductivity in twisted bilayer graphene (TBG) can be explained as a consequence of the Kohn-Luttinger (KL) instability which leads to an effective attraction between electrons with originally repulsive interaction. Usually, the KL instability takes place at extremely low energy scales, but in TBG, a doubling and subsequent strong coupling of the van Hove singularities (vHS) in the electronic spectrum occurs as the magic angle is approached, leading to extended saddle points in the highest valence band with almost perfect nesting between states belonging to different valleys. The highly anisotropic screening induces an effective attraction in a $p$-wave channel with odd parity under the exchange of the two disjoined patches of the Fermi line. We also predict the appearance of a spin-density wave instability, adjacent to the superconducting phase, and the opening of a gap in the electronic spectrum from the condensation of spins with wave vector corresponding to the nesting vector close to the vHS.
\end{abstract}

DOI: 10.1103/PhysRevLett.122.026801

Introduction.-The discovery of superconductivity [1] in twisted bilayer graphene (TBG) with a critical temperature of $1.7 \mathrm{~K}$ at small twist angles around $1.1^{\circ}$ and moiré period of $\sim 13.5 \mathrm{~nm}$ might be the missing puzzle needed to resolve long-standing questions related to high- $T_{c}$ superconductivity in layered compounds [2-4]. This hope is based on the fact that the phase diagram of TBG is characterized by a Mott insulator at half filling of the highest valence band (VB), corresponding to two electrons per Moiré unit cell, which upon doping turns into a superconducting (SC) instability [5]. Increasing structural instead of chemical complexity can thus provide an alternative route to design devices with novel functionalities and therefore TBG has attracted considerable interest even before the publication of Refs. [1,5] due to its novel electronic [6-16], optical [17-22], and plasmonic [23-26] properties.

Although the findings by Jarillo-Herrero and co-workers have attracted immense attention [16,27-51], only very few attempts have focused on identifying the driving force of the superconductivity at the so-called magic twist angle $\theta_{m} \approx 1.05^{\circ}$, where the highest VB becomes extremely flat [52-54]. One predictable theory was discussed in Ref. [44], which sets the electron-phonon interaction as the basis of the pairing mechanism. On the other hand, the strong correlations that develop near the magic angle leave also room for the less conventional possibility of a purely electronic mechanism of superconductivity, following a route which has been also explored in the context of monolayer graphene [55-63]. In this respect, there have been a couple of proposals in Refs. [42,43] focusing on that kind of approach from a microscopic standpoint, investigating the weak-coupling instabilities arising from the shape of the Fermi surface (although for twisted bilayers relatively far away from the magic angle).

In this Letter, we unveil what may be the key interaction governing the superconductivity of TBG, identifying for that purpose a number of universal topological features in
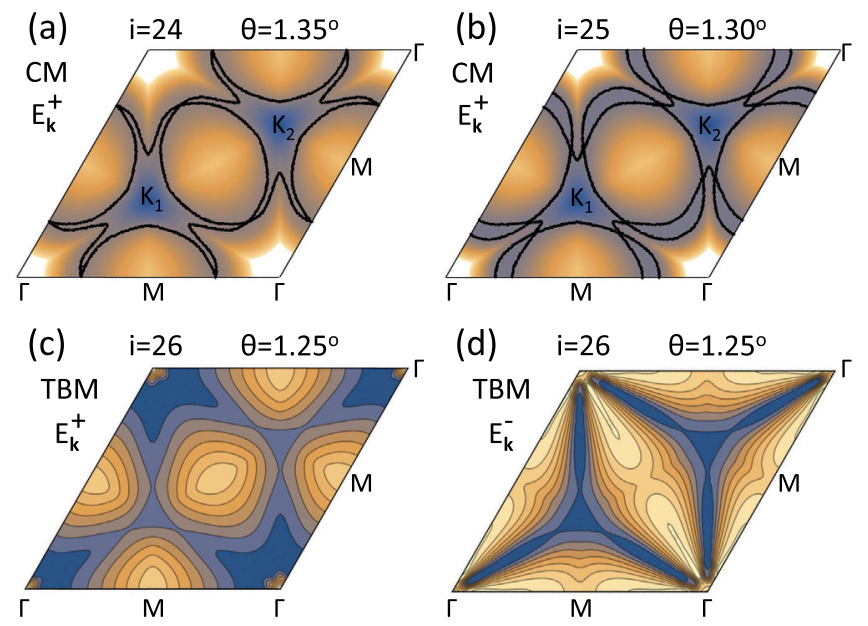

FIG. 1. (a) and (b) Density plot of the energy dispersion of the highest valence band $E_{k}^{+}=\max \left(E_{k}^{K}, E_{k}^{K^{\prime}}\right)$ in the moiré Brillouin zone of the continuous model for two different twist angles. Dark (bright) colors represent high (low) energies and the black contour lines represent the Fermi surface at the energy of the van Hove singularity $E_{\mathrm{vH}}$. There occurs a doubling of the vHS at some critical angle $\theta_{i=24}>\theta_{c}^{+}>\theta_{i=25}$, i.e., for lower $\theta$ there are twelve saddle points located inside the MBZ close to the lines that connect the $\Gamma$ and $K_{\ell}$ points. (c) and (d) Contour plot of the highest valence bands $E_{k}^{+}$and $E_{k}^{-}$of the tight-binding model for $i=26$. The two sets of vHS belonging to different valleys have already merged and are found now in different bands $E_{k}^{+}$and $E_{k}^{-}$. 
the electronic dispersion of the highest VB which are indispensable to understand the pairing mechanism at the microscopic level. We show that, below a certain critical twist angle $\theta_{c}^{-} \approx 1.3^{\circ}$, there is both a doubling and strong coupling of the van Hove singularities (vHS) in the electronic spectrum, leading to extended saddle points in the highest VB with almost perfect nesting between states belonging to different valleys. This induces a highly anisotropic screening of the Coulomb interaction, leading necessarily to an effective attractive interaction in a channel with $p$-wave symmetry which is the seed required to trigger the superconducting instability.

Our theoretical construction constitutes a variant of the so-called Kohn-Luttinger (KL) mechanism [64,65], which was proposed as a route to develop a superconducting instability starting from a purely repulsive interaction. We thus put forward a microscopic theory of superconductivity in TBG which only relies on the Coulomb interaction, giving definite quantitative predictions for the critical energy scale of the superconducting transition in a range which spans from weak coupling up to a much stronger instability depending on the proximity of the Fermi level to the vHS. We also complete the study by discussing the spin-density wave (SDW) adjacent to the superconducting phase, and whose onset takes place typically for a critical Coulomb interaction which is below the bandwidth of the highest VB, thus reassuring our microscopic approach to the superconductivity of TBG.

Models.-To model TBG, we will use the continuous model (CM) that treats commensurate lattices parametrized by the integer $i$ with the twist angle $\cos \theta_{i}=$ $\left(3 i^{2}+3 i+0.5\right) /\left(3 i^{2}+3 i+1\right)[12,53,66,67]$. For these angles, we will also use the tight-binding model (TBM) of TBG $[9,17,52]$, which already incorporates the coupling between states around the $K$ and $K^{\prime}$ valley. In the Supplemental Material [68], the real space image and the Brillouin zones of the two layers are shown together with the moire Brillouin zone (MBZ) around the two valleys $K$ and $K^{\prime}$.

In the TBM, the highest VB containing up to four electrons (corresponding to twofold spin and valley degeneracy as mentioned in the introduction) thus splits in two bands. Consequently, the TBM description can be compared to the CM by combining in the latter the highest VB corresponding to each $K$ point, $E_{k}^{K}$ and $E_{k}^{K^{\prime}}$, to $E_{k}^{+}=$ $\max \left(E_{k}^{K}, E_{k}^{K^{\prime}}\right)$ and $E_{k}^{-}=\min \left(E_{k}^{K}, E_{k}^{K^{\prime}}\right)$. The result of the comparison turns out to be in general quite satisfactory, as shown in the Supplemental Material [68]. For twist angles $\theta>1.1^{\circ}$, the two combined bands $E_{k}^{+}$and $E_{k}^{-}$are only degenerate on the six $\Gamma K_{\ell}$ lines for which $E_{k}^{K}=E_{k}^{K^{\prime}}$ in the $\mathrm{CM}, K_{\ell}$ being the Dirac point belonging to layer $\ell=1,2$ and $\boldsymbol{k}$ measured with respect to the corresponding valley. Also the density plot of the highest VB and degeneracy contours for smaller twist angles $\theta<1.1^{\circ}$ are discussed in the Supplemental Material [68]. van Hove singularities in the highest VBs.-The KL mechanism we are proposing relies on the anisotropic screening that can be provided by a strong vHS, induced by a large number of saddle points within the MBZ which is crucial to tip the scale towards a SC instability [61]. In this regard, the highest $\mathrm{VB} E_{k}^{+}$witnesses important changes with respect to its topology as function of the twist angle. At large twist angles, there are six vHS (saddle points), three for each valley and located around the three $M$ points of the MBZ. Decreasing the twist angle, the vHS move away from the $M$ points and for $i \approx 24-25$, we observe a splitting of the saddle points, see Figs. 1(a) and 1(b), where the density plot of $E_{k}^{+}$is shown together with the Fermi line at the vHS. We thus identify a first critical angle $\theta_{c}^{+}$, where a doubling of vHS occurs from six to twelve. The exact crossing point usually occurs at a noncommensurate critical angle $\theta_{c}^{+}$that can be treated by more advanced numerical techniques [76,77].

At smaller twist angles $\theta<\theta_{c}^{+}$, the evolution of the saddle points critically depends on the coupling between states at different $K$ and $K^{\prime}$ valleys, best captured by the TBM. Decreasing the twist angle, the pairs of vHS move closer to the $\Gamma K_{\ell}$ lines up to a second critical angle $\theta_{c}^{-}$at which the two saddle points of the $E_{k}^{+}$band merge and a new saddle point in the $E_{k}^{-}$band emerges. Both vHS are then pinned to the $\Gamma K_{\ell}$ line, see Figs. 1(c) and 1(d), where the contour plot of $E_{k}^{+}$and $E_{k}^{-}$is shown for $i=26$.

The splitting of the six pairs of vHS has two important consequences. First, the overlap between the states around the saddle points becomes approximately one due to the direct coupling of the two valleys; second, the vHS become further extended. Both consequences lead to a large susceptibility of particle-hole pairs when the Fermi energy is close to the vHS, which can trigger the SC instability via the KL mechanism.

Kohn-Luttinger instability.-The KL instability can be analyzed starting from a conventional BCS approach where the Cooper-pair vertex $V$ is parametrized in terms of the angles $\phi$ and $\phi^{\prime}$ of the respective momenta of the spin-up incoming and outgoing electrons on each contour line of energy $\varepsilon$. The iteration of the scattering between the electrons in the Cooper pair can be encoded in the selfconsistent equation

$$
\begin{aligned}
V\left(\phi, \phi^{\prime}\right)= & V_{0}\left(\phi, \phi^{\prime}\right)-\frac{1}{(2 \pi)^{2}} \int^{\Lambda_{0}} \frac{d \varepsilon}{\varepsilon} \\
& \times \int_{0}^{2 \pi} d \phi^{\prime \prime} \frac{\partial k_{\perp}}{\partial \varepsilon} \frac{\partial k_{\|}}{\partial \phi^{\prime \prime}} V_{0}\left(\phi, \phi^{\prime \prime}\right) V\left(\phi^{\prime \prime}, \phi^{\prime}\right),
\end{aligned}
$$

where $k_{\|}, k_{\perp}$ are the respective longitudinal and transverse components of the momentum while $V_{0}\left(\phi, \phi^{\prime}\right)$ stands for the bare vertex at a high-energy cutoff $\Lambda_{0}$. Differentiating Eq. (1) with respect to the cutoff, we end up with the scaling equation 


$$
\Lambda \frac{\partial \hat{V}\left(\phi, \phi^{\prime}\right)}{\partial \Lambda}=\frac{1}{2 \pi} \int_{0}^{2 \pi} d \phi^{\prime \prime} \hat{V}\left(\phi, \phi^{\prime \prime}\right) \hat{V}\left(\phi^{\prime \prime}, \phi^{\prime}\right)
$$

where $\quad \hat{V}\left(\phi, \phi^{\prime}\right)=F(\phi) F\left(\phi^{\prime}\right) V\left(\phi, \phi^{\prime}\right) \quad$ and $\quad F(\phi)=$ $\sqrt{\left(\partial k_{\perp} / \partial \varepsilon\right)\left(\partial k_{\|} / \partial \phi\right) / 2 \pi}$. It is clear that, if $\hat{V}\left(\phi, \phi^{\prime}\right)$ has some negative eigenvalue at the high-energy regime of $\Lambda$, this will result in a divergent growth of the BCS vertex in the low-energy limit $\Lambda \rightarrow 0$, which is the signature of a pairing instability.

The KL mechanism of superconductivity is enhanced for electron systems in which the Fermi velocity has a large anisotropy along the Fermi line. The anisotropic screening induced by particle-hole excitations gives rise to the angular dependence of the BCS vertex which, assuming a constant interaction $U$ in momentum space [68], becomes in the random-phase approximation (RPA) [78]

$$
V_{0}\left(\phi, \phi^{\prime}\right)=U+\frac{U^{2} \chi_{k+k^{\prime}}}{1-U \chi_{k+k^{\prime}}}+\frac{U^{3} \chi_{k-k^{\prime}}^{2}}{1-U^{2} \chi_{k-k^{\prime}}^{2}},
$$

where $\boldsymbol{k}, \boldsymbol{k}^{\prime}$ are the respective momenta at angles $\phi, \phi^{\prime}$ and $\chi_{q}$ is the particle-hole susceptibility at momentum transfer $\boldsymbol{q}$.

A simple argument allows us to understand why there is always an effective attractive interaction in TBG approaching the magic angle: since the Fermi line near the vHS in the VB $E_{k}^{-}$consists of two disjoined patches, the two electrons forming the Cooper pair belong to different patches, see Fig. 2(b). We can now distinguish between two different contributions to the BCS vertex, depending on whether the electrons of the Cooper pair scatter within the same patch of the Fermi line (intrapatch vertex $V_{\text {intra }}$ ) or whether they scatter exchanging their patches (interpatch vertex $\left.V_{\text {inter }}\right)$. Close to the vHS of the VB $E_{k}^{-}$, the particlehole susceptibility has a large peak at small momentum transfer [as seen in the inset of Fig. 2(a)], which leads to a strong enhancement of the second term on the right-hand side of Eq. (3) for $V_{\text {inter }}$, when $\boldsymbol{k} \approx-\boldsymbol{k}^{\prime}$. This enhanced susceptibility can be understood from the almost perfect nesting condition that connects the two opposite lines of the three side lobes of each patch, as depicted in Fig. 2(b). In the case of $V_{\text {intra }}$, however, the enhancement corresponds to the third term in the equation when $\boldsymbol{k} \approx \boldsymbol{k}^{\prime}$, which is smaller than the contribution that comes from $V_{\text {inter }}$, as shown in Fig. 2(a).

The full BCS vertex $\hat{V}\left(\phi, \phi^{\prime}\right)$ now becomes a matrix such that

$$
\hat{V}=\left(\begin{array}{ll}
\hat{V}_{\text {intra }} & \hat{V}_{\text {inter }} \\
\hat{V}_{\text {inter }} & \hat{V}_{\text {intra }}
\end{array}\right) .
$$

Given that the interpatch scattering is in general more intense than the intrapatch interaction, $\hat{V}_{\text {inter }} \gtrsim \hat{V}_{\text {intra }}$, we (a)

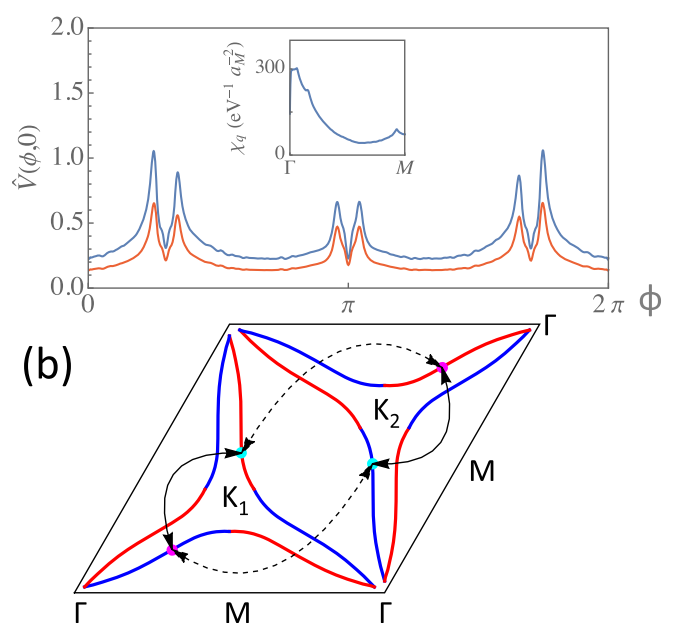

FIG. 2. (a) Plot of the BCS vertices $\hat{V}_{\text {inter }}(\phi, 0)$ (blue curve) and $\hat{V}_{\text {intra }}(\phi, 0)$ (red curve) for angle $\phi$ running along one of the patches of the Fermi line of a twisted bilayer with $i=26$ and Fermi level placed $0.1 \mathrm{meV}$ below the vHS of the valence band $E_{k}^{-}$, for a value of the Coulomb interaction $U / a_{M}^{2}=2 \mathrm{meV}\left(a_{M}\right.$ being the Moiré lattice constant of the twisted bilayer graphene). The inset shows the particle-hole susceptibility $\chi_{q}$ (in units of $\mathrm{eV}^{-1} a_{M}^{-2}$ ) for momenta running from $\Gamma$ to $\mathrm{M}$ in the MBZ with the same parameters. (b) Intrapatch (solid lines) and interpatch (dashed lines) scattering processes of two Cooper pairs (cyan and magenta dots). The blue, respectively, red curves indicate the states belonging to the two different valleys of the Fermi line $0.2 \mathrm{meV}$ below the energy of the vHS of $E_{k}^{-}$as obtained from the CM with $i=29$.

find an attractive channel with negative eigenvalue and antisymmetric amplitude in the two disjoined patches of the Fermi line; see also the Supplemental Material [68].

Broken symmetry phases. - The poles in the RPA expression in Eq. (3) imply the existence of a critical interaction strength at which the BCS vertex as well as other response functions diverge, indicating the trend towards broken symmetry phases in the electronic system. The competition between these low-energy phases can be analyzed in an unbiased manner by means of a renormalization group (RG) approach, see Supplemental Material [68]. It turns out that there is a phase boundary between the pairing instability and a spin-density wave instability which prevails above a critical interaction $U_{c}$, as shown in Fig. 3(a). The value of $U_{c}$ is dictated by the peak of the particle-hole susceptibility, $\chi_{q}$, whose position in momentum space sets the wave vector of the spin-density wave instability-as seen in the inset of Fig. 2(a). We expect that in the strong coupling regime, the spin-density wave instability, which also opens a gap in the electronic spectrum [74,75], should correspond to the insulating phase observed in the experiments of Ref. [1]. Nevertheless, we stress that the KL instability is always dominant before reaching the critical interaction $U_{c}$, as it only relies on the anisotropy of the $\mathrm{BCS}$ vertex. 
(a)

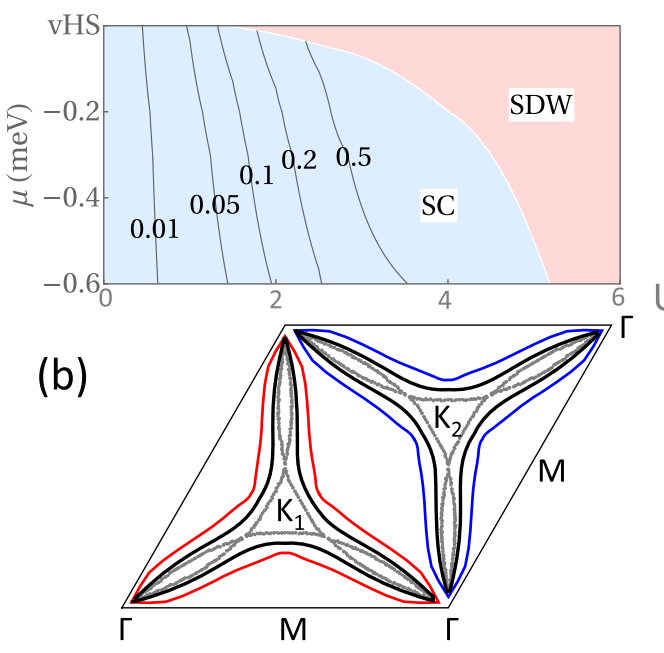

FIG. 3. (a) Phase diagram as function of the chemical potential $\mu$ relative to the energy at the vHS of $E_{k}^{-}$and the bare interaction $U$ (in units of $\mathrm{meV} a_{M}^{2}$ ). The superconducting instability (SC) always precedes the spin-density-wave instability (SDW). Contour lines refer to the largest value of the BCS coupling $|\lambda|$ in the channel with dominant attractive interaction. (b) Gap structure with $\Delta_{\phi}=\Delta_{0}[0.75-0.25 \cos (3 \phi)]$ as red and blue curves to indicate the sign change under parity. The gap of $\Delta_{0}=8 \mathrm{~K}$ is exaggerated in order to demonstrate the qualitative behavior, i.e., the gap region is larger around regions where screening is enhanced. Also shown the Fermi line at the vHS (gray dots) and the Fermi line $0.2 \mathrm{meV}$ below the energy of the vHS (black).

In our RG approach, $U$ corresponds to the interaction potential at zero momentum, i.e., the scale-invariant part of the interaction close to the vHS, see Supplemental Material [68]. As shown in Fig. 3(a), the values of $U_{c}$ are of the order of a few meV (times the square of the moiré lattice constant $\left.a_{M}\right)$. Those values match well with the order of magnitude expected for the Coulomb repulsion, which must undergo a reduction (with respect to that in monolayer graphene) by a factor inversely proportional to the number of atoms in the unit cell of the twisted bilayer. The values of $U / a_{M}^{2}$ needed to trigger the broken symmetry phase (and thus a preceding KL instability) are therefore below the bandwidth $W \sim$ $5 \mathrm{meV}$ of the highest VB of the twisted bilayers considered here $(i=26)$.

Superconducting order parameter.-Fourier transforming the BCS vertex, we can identify the attractive channels with negative eigenvalues $\lambda$ and their respective symmetries. We recall that, for each disjoined patch of the Fermi line with $K_{\ell}$ as its center point, the relevant point group is $C_{3 v}$. The irreducible representations of $C_{3 v}$ can be characterized by the Fourier components, i.e., $A_{1} \rightarrow[\cos (3 n \phi)], A_{2} \rightarrow[\sin (3 n \phi)]$, and $E \rightarrow[\cos (m \phi)$, $\sin (m \phi)]$ with $m \neq 3 n, \phi=0$ corresponding to the point on the Fermi line closer to $K_{\ell}$.

Interestingly, we find attractive channels belonging to all three irreducible representations with odd and even parity, see Table I. But the dominant instability is given by an
TABLE I. Most negative (attractive) eigenvalues and their respective irreducible representations of $C_{3 v}$ for $U / a_{M}^{2}=$ $2.5 \mathrm{meV}$ and the Fermi level placed $0.1 \mathrm{meV}$ below the vHS in the valence band $E_{k}^{-}$.

\begin{tabular}{lcl}
\hline \hline Eigenvalue $\lambda$ & Irreducible representation & Parity \\
\hline-0.51 & $A_{1}$ & Odd \\
-0.11 & $E$ & Odd \\
-0.10 & $A_{2}$ & Even \\
-0.08 & $A_{1}$ & Odd \\
-0.06 & $E$ & Even \\
\hline \hline
\end{tabular}

order parameter that transforms according to $A_{1}$ with odd parity and can be approximated by $\Delta_{\phi}=\Delta_{0}[0.75-$ $0.25 \cos (\phi)]$. The values of the most attractive coupling $|\lambda|$ are represented in Fig. 3(a). The scale of the gap $\Delta_{0}$ is obtained by solving Eq. (2) and yields

$$
\Delta_{0}=\Lambda_{0} \exp (-1 /|\lambda|)
$$

We can approximate the cutoff scale $\Lambda_{0}$ by the separation of the Fermi line from the energy of the vHS, i.e., $\Lambda_{0} \sim$ $0.1 \mathrm{meV}$ which is of the order of $1 \mathrm{~K}$. The gap structure is shown in Fig. 3(b) for $\Delta_{0}=8 \mathrm{~K}$. The order parameter is slightly suppressed close to the $K_{\ell}$ points and changes sign under parity indicated by the blue and red lines, respectively. Also shown is the Fermi line $0.2 \mathrm{meV}$ below the $E_{\mathrm{vH}}$ (black) as well as the Fermi line at the vHS containing the six saddle points (gray dots).

Summary.-We present a quantitative theory for the recently discovered superconductivity in twisted bilayer graphene close to, but not at the first magic angle. Our theory rests upon the observation that there is a saddlepoint splitting at some critical twist angle which induces strong intervalley coupling. Fermi lines close to the vHS are disjunct and display regions of almost perfect nesting giving rise to a large susceptibility for small wave numbers and thus to an enhanced Kohn-Luttinger instability. The dominant instability yields an order parameter with odd parity that has an approximate $s$-wave symmetry around the two patches of the Fermi line, i.e., it is spin-triplet and valley-singlet. The sign-change of the superconducting gap for different valleys should be detectable via STM by measuring the quasiparticle interferences. Furthermore, our theory predicts a scale of the superconducting gap which agrees with the experimental findings of Ref. [1].

Let us finally address open questions. In order to find the superconducting instability in the middle of the VB, the level of the vHS should correspond to half-filling. This is indeed the case for angles in the vicinity (but not at) the magic angle and there is further experimental evidence of an interaction induced pinning of the vHS to half filling [79,80], see Supplemental Material [68]. For twist angles in the immediate vicinity of the magic angle, our computational scheme breaks down there, but we believe 
that the key features we have found driving the KL mechanism must also be present in that regime, possibly yielding an even larger superconducting gap.

We thank G. Gómez-Santos and H. Kohler for useful discussions. This work has been supported by Spain's MINECO under Grants No. FIS2017-82260-P and No. FIS2014-57432-P.

[1] Y. Cao, V. Fatemi, S. Fang, K. Watanabe, T. Taniguchi, E. Kaxiras, and P. Jarillo-Herrero, Nature (London) 556, 43 (2018).

[2] R. Micnas, J. Ranninger, and S. Robaszkiewicz, Rev. Mod. Phys. 62, 113 (1990).

[3] P. A. Lee, N. Nagaosa, and X.-G. Wen, Rev. Mod. Phys. 78, 17 (2006).

[4] G. R. Stewart, Rev. Mod. Phys. 83, 1589 (2011).

[5] Y. Cao, V. Fatemi, A. Demir, S. Fang, S. L. Tomarken, J. Y. Luo, J. D. Sanchez-Yamagishi, K. Watanabe, T. Taniguchi, E. Kaxiras, R. C. Ashoori, and P. Jarillo-Herrero, Nature (London) 556, 80 (2018).

[6] S. Shallcross, S. Sharma, and O. A. Pankratov, Phys. Rev. Lett. 101, 056803 (2008).

[7] H. Schmidt, T. Lüdtke, P. Barthold, and R. J. Haug, Phys. Rev. B 81, 121403 (2010).

[8] G. Li, A. Luican, J. M. B. Lopes dos Santos, A. H. Castro Neto, A. Reina, J. Kong, and E. Y. Andrei, Nat. Phys. 6, 109 (2010).

[9] G. T. de Laissardière, D. Mayou, and L. Magaud, Nano Lett. 10, 804 (2010).

[10] D. S. Lee, C. Riedl, T. Beringer, A. H. Castro Neto, K. von Klitzing, U. Starke, and J. H. Smet, Phys. Rev. Lett. 107, 216602 (2011)

[11] A. Luican, G. Li, A. Reina, J. Kong, R. R. Nair, K. S. Novoselov, A. K. Geim, and E. Y. Andrei, Phys. Rev. Lett. 106, 126802 (2011).

[12] P. Moon and M. Koshino, Phys. Rev. B 85, 195458 (2012).

[13] J. D. Sanchez-Yamagishi, T. Taychatanapat, K. Watanabe, T. Taniguchi, A. Yacoby, and P. Jarillo-Herrero, Phys. Rev. Lett. 108, 076601 (2012).

[14] I. Brihuega, P. Mallet, H. González-Herrero, G. Trambly de Laissardière, M. M. Ugeda, L. Magaud, J. M. GómezRodríguez, F. Ynduráin, and J.-Y. Veuillen, Phys. Rev. Lett. 109, 196802 (2012).

[15] K. Kim, A. DaSilva, S. Huang, B. Fallahazad, S. Larentis, T. Taniguchi, K. Watanabe, B. J. LeRoy, A. H. MacDonald, and E. Tutuc, Proc. Natl. Acad. Sci. U.S.A.114, 3364 (2017).

[16] Y.-H. Zhang, D. Mao, Y. Cao, P. Jarillo-Herrero, and T. Senthil, arXiv:1805.08232.

[17] P. Moon and M. Koshino, Phys. Rev. B 87, 205404 (2013).

[18] R. W. Havener, Y. Liang, L. Brown, L. Yang, and J. Park, Nano Lett. 14, 3353 (2014).

[19] H. Patel, R. W. Havener, L. Brown, Y. Liang, L. Yang, J. Park, and M. W. Graham, Nano Lett. 15, 5932 (2015).

[20] C.-J. Kim, S.-C. A., Z. Ziegler, Y. Ogawa, C. Noguez, and J. Park, Nat. Nanotechnol. 11, 520 (2016).

[21] E. S. Morell, L. Chico, and L. Brey, 2D Mater. 4, 035015 (2017).
[22] T. Stauber, T. Low, and G. Gómez-Santos, Phys. Rev. Lett. 120, 046801 (2018).

[23] T. Stauber, P. San-Jose, and L. Brey, New J. Phys. 15, 113050 (2013).

[24] T. Stauber and H. Kohler, Nano Lett. 16, 6844 (2016).

[25] F. Hu, S. R. Das, Y. Luan, T.-F. Chung, Y. P. Chen, and Z. Fei, Phys. Rev. Lett. 119, 247402 (2017).

[26] S. S. Sunku, G. X. Ni, B. Y. Jiang, H. Yoo, A. Sternbach, A. S. McLeod, T. Stauber, L. Xiong, T. Taniguchi, K. Watanabe, P. Kim, M. M. Fogler, and D. N. Basov, Science 362, 1153 (2018).

[27] C. Xu and L. Balents, Phys. Rev. Lett. 121, 087001 (2018).

[28] G. E. Volovik, JETP Lett. 107, 516 (2018).

[29] N. F. Q. Yuan and L. Fu, Phys. Rev. B 98, 045103 (2018).

[30] H. C. Po, L. Zou, A. Vishwanath, and T. Senthil, Phys. Rev. X 8, 031089 (2018).

[31] B. Roy and V. Juricic, arXiv:1803.11190.

[32] H. Guo, X. Zhu, S. Feng, and R. T. Scalettar, Phys. Rev. B 97, 235453 (2018).

[33] J. F. Dodaro, S. A. Kivelson, Y. Schattner, X. Q. Sun, and C. Wang, Phys. Rev. B 98, 075154 (2018).

[34] G. Baskaran, arXiv:1804.00627.

[35] C.-C. Liu, L.-D. Zhang, W.-Q. Chen, and F. Yang, Phys. Rev. Lett. 121, 217001 (2018).

[36] T. Huang, L. Zhang, and T. Ma, arXiv:1804.06096.

[37] K. Slagle and Y. B. Kim, arXiv:1805.05331.

[38] T. J. Peltonen, R. Ojajrvi, and T. T. Heikkil, Phys. Rev. B 98, 220504 (2018)

[39] D. M. Kennes, J. Lischner, and C. Karrasch, Phys. Rev. B 98, 241407(R) (2018).

[40] M. Koshino, N. F. Q. Yuan, T. Koretsune, M. Ochi, K. Kuroki, and L. Fu, Phys. Rev. X 8, 031087 (2018).

[41] J. Kang and O. Vafek, Phys. Rev. X 8, 031088 (2018).

[42] H. Isobe, N. F. Q. Yuan, and L. Fu, Phys. Rev. X 8, 041041 (2018).

[43] Y.-Z. You and A. Vishwanath, arXiv:1805.06867.

[44] F. Wu, A. H. MacDonald, and I. Martin, Phys. Rev. Lett. 121, 257001 (2018).

[45] J. M. Pizarro, M. J. Calderón, and E. Bascones, arXiv:1805 .07303

[46] H. K. Pal, arXiv:1805.08803.

[47] M. Ochi, M. Koshino, and K. Kuroki, Phys. Rev. B 98, 081102 (2018).

[48] A. Thomson, S. Chatterjee, S. Sachdev, and M. S. Scheurer, Phys. Rev. B 98, 075109 (2018).

[49] S. Carr, S. Fang, P. Jarillo-Herrero, and E. Kaxiras, Phys. Rev. B 98, 085144 (2018).

[50] F. Guinea and N. R. Walet, Proc. Natl. Acad. Sci. U.S.A. 115, 13174 (2018).

[51] L. Zou, H. C. Po, A. Vishwanath, and T. Senthil, Phys. Rev. B 98, 085435 (2018).

[52] E. Suárez Morell, J. D. Correa, P. Vargas, M. Pacheco, and Z. Barticevic, Phys. Rev. B 82, 121407 (2010).

[53] R. Bistritzer and A. H. MacDonald, Proc. Natl. Acad. Sci. U.S.A. 108, 12233 (2011).

[54] P. San-Jose, J. González, and F. Guinea, Phys. Rev. Lett. 108, 216802 (2012)

[55] B. Uchoa and A. H. Castro Neto, Phys. Rev. Lett. 98, 146801 (2007). 
[56] A. M. Black-Schaffer and S. Doniach, Phys. Rev. B 75, 134512 (2007).

[57] J. González, Phys. Rev. B 78, 205431 (2008).

[58] C. Honerkamp, Phys. Rev. Lett. 100, 146404 (2008).

[59] B. Roy and I. F. Herbut, Phys. Rev. B 82, 035429 (2010).

[60] J. L. McChesney, A. Bostwick, T. Ohta, T. Seyller, K. Horn, J. González, and E. Rotenberg, Phys. Rev. Lett. 104, 136803 (2010).

[61] R. Nandkishore, L. S. Levitov, and A. V. Chubukov, Nat. Phys. 8, 158 (2012).

[62] F. Guinea and B. Uchoa, Phys. Rev. B 86, 134521 (2012).

[63] J. González, Phys. Rev. B 88, 125434 (2013).

[64] W. Kohn and J. M. Luttinger, Phys. Rev. Lett. 15, 524 (1965).

[65] M. A. Baranov, A. V. Chubukov, and M. Yu. Kagan, Int. J. Mod. Phys. B 06, 2471 (1992).

[66] J. M. B. Lopes dos Santos, N. M. R. Peres, and A. H. Castro Neto, Phys. Rev. Lett. 99, 256802 (2007).

[67] E. J. Mele, Phys. Rev. B 81, 161405 (2010).

[68] See Supplemental Material at http://link.aps.org/ supplemental/10.1103/PhysRevLett.122.026801 for more details and additional numerical results, which includes Refs. [69-75].
[69] T. Stauber, T. Low, and G. Gómez-Santos, Phys. Rev. B 98, 195414 (2018).

[70] N. N. T. Nam and M. Koshino, Phys. Rev. B 96, 075311 (2017).

[71] J. González, F. Guinea, and M. Vozmediano, Nucl. Phys. B485, 694 (1997).

[72] T. Stauber, Phys. Rev. B 82, 201404 (2010).

[73] J. Alvarez, J. González, F. Guinea, and M. A. H. Vozmediano, J. Phys. Soc. Jpn. 67, 1868 (1998).

[74] J. González, Nucl. Phys. B642, 407 (2002).

[75] J. González, Phys. Rev. B 67, 054510 (2003).

[76] S. Carr, D. Massatt, S. Fang, P. Cazeaux, M. Luskin, and E. Kaxiras, Phys. Rev. B 95, 075420 (2017).

[77] E. Cancès, P. Cazeaux, and M. Luskin, J. Math. Phys. (N.Y.) 58, 063502 (2017).

[78] D. J. Scalapino, E. Loh, and J. E. Hirsch, Phys. Rev. B 35, 6694 (1987).

[79] Y. Kim, P. Herlinger, P. Moon, M. Koshino, T. Taniguchi, K. Watanabe, and J. H. Smet, Nano Lett. 16, 5053 (2016).

[80] Y. Cao, J. Y. Luo, V. Fatemi, S. Fang, J. D. SanchezYamagishi, K. Watanabe, T. Taniguchi, E. Kaxiras, and P. Jarillo-Herrero, Phys. Rev. Lett. 117, 116804 (2016). 